\title{
System Support Mapping
}

National Cancer Institute

\section{Source}

National Cancer Institute. System Support Mapping. NCI Thesaurus. Code C148263.

A method by which trained facilitators guide participants to create a visual diagram of their self-management activities within their broader life context. MAP helps participants tang ibly see complex self-manag ement activities on paper, facilitating self-awareness and patient-driven eng agement which makes them more actionable. 